\title{
PROPERTIES OF EMERGING BIPOLAR ACTIVE REGIONS
}

\author{
KAREN L. HARVEY 1 \\ Solar Physics Research Corporation, 4720 Calle Desecada, \\ Tucson, AZ 85718, U.S.A.
}

\begin{abstract}
Characteristics and the emergence pattern of bipolar active regions are investigated using NSO/KP full disk magnetograms. Discussed in this paper are two samples of newly emerged bipolar regions: 9492 ephemeral regions and 978 active regions. Their distributions in size, latitude, and orientation are compared. It is concluded that ephemeral regions are the small scale end of a wide spectrum of active regions.
\end{abstract}

\section{INTRODUCTION}

This paper presents a summary of some results of a comparison of the distributions in size, latitude, and orientation of the magnetic axes of active regions and ephemeral regions (discussed in detail in Harvey and Zwaan 1993, Harvey 1993).

Daily magnetograms made at the NSO/KP are used to identify emerging bipolar regions during 29 solar rotations selected from the 1975 to 1986. Area is chosen as a measure of the magnitude of these regions. Two samples of bipoles are studied: 978 active regions, ranging in size from 120 to $3550 \mu \mathrm{H}_{\odot}$ and 9492 ephemeral regions, ranging in size from 5 to $120 \mu \mathrm{H}_{\odot} .\left(\mu \mathrm{H}_{\odot}\right.$ is the area in millionths of a solar hemisphere.)

Characteristics of these regions are investigated using specific criteria to define an active region and an ephemeral region. Considered are only active regions that appear as strictly bipolar magnetic entities and emerge completely on the visible hemisphere. The identification of an ephemeral region is based on a detailed comparison of two successive daily magnetograms and the selection on the second day of those new bipolar structures that cannot be explained by the chance encounter of opposite polarity magnetic network elements. Each selected bipole was included only once in these analyses.

Region counts are corrected for the reduction of visibility of such regions near the solar limb, for data gaps, for regions lost because of the restriction that the maximum development be observed, and for the reduced visibility of smaller active regions (areas 120-460 $\mu \mathrm{H}_{\odot}$ ) emerging within the boundaries of existing active regions. These corrections are relevant and necessary for the

\footnotetext{
${ }^{1}$ Visitor, National Solar Observatory, National Optical Astronomy Observatories, Operated by the Association of Universities for Research in Astronomy, Inc., under Cooperative Agreement with the National Science Foundation.
} 


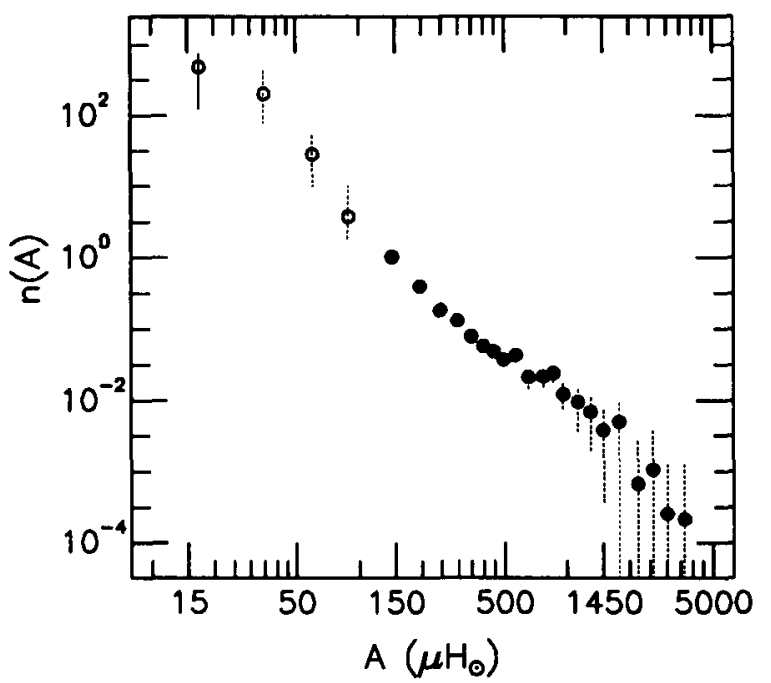

Fig. 1. Size distribution, $\mathrm{n}(\mathrm{A})$, for active regions (•) and for ephemeral regions $(0)$. Vertical lines indicate the estimated error.

determination of the actual distribution of sizes of active regions over the large range considered in this study.

\section{SIZE DISTRIBUTION}

Figure 1 compares the size distributions, $n(A)$, i.e. the number of regions per day and per region area bin of $48 \mu \mathrm{H}_{\odot}$, of active regions and of ephemeral regions. These two samples form a smooth and continuous distribution from the largest active regions to the smallest ephemeral regions, increasing sharply with decreasing region size.

\section{LATITUDE DISTRIBUTION}

During a cycle, activity moves progressively toward the equator. To eliminate this time-varying component, the latitude distribution for the 978 active regions and 9492 ephemeral regions was determined relative to the average latitude of regions larger than $315 \mu \mathrm{H}_{\odot}$ and of an additional sample of 1413 sunspot regions with sunspots of total area greater than $100 \mu \mathrm{H}_{\odot}$ (from Solar Geophysical Data). As shown in Figure 2, for regions of all size ranges including ephemeral regions, the skewed latitude distributions peak at the average region latitude. Active regions larger than $315 \mu \mathrm{H}_{\odot}$ emerge within a confined, relatively narrow range of latitudes. The distribution in latitude expands to a progressively and significantly wider range for smaller regions $\left(5-315 \mu \mathrm{H}_{\odot}\right)$, reaching its maximum extent for the ephemeral regions. 


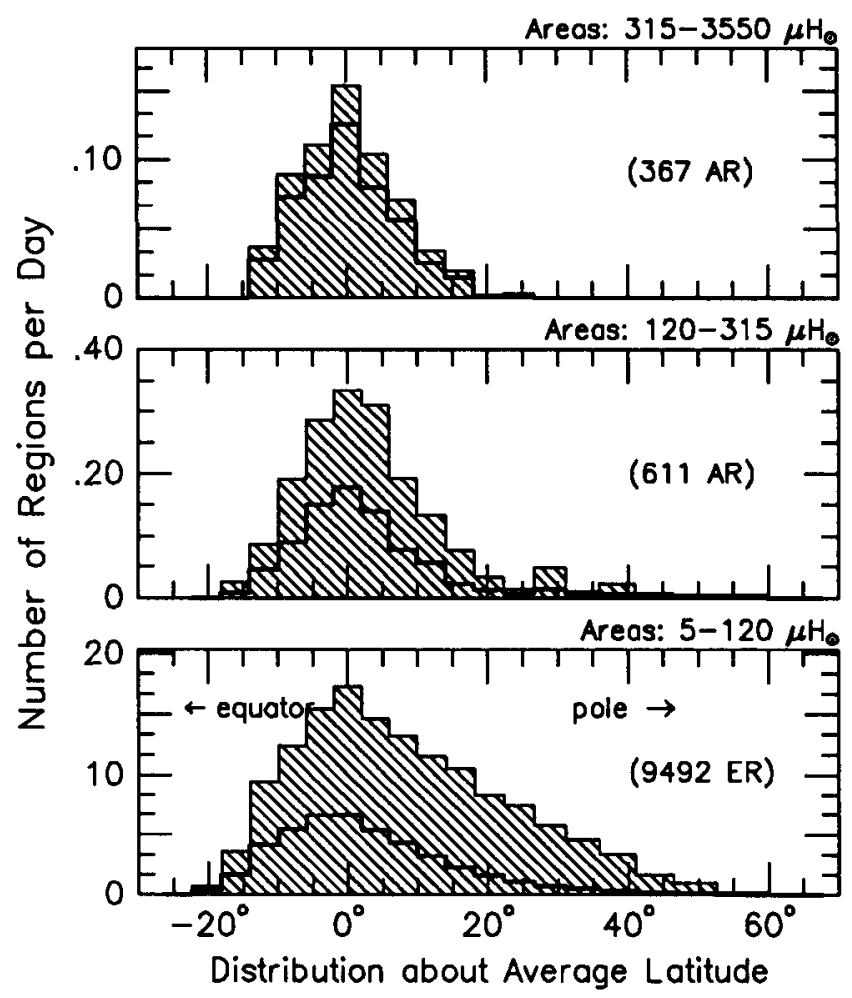

Fig. 2. Latitude distribution of ephemeral regions and active regions about the average latitude of active regions larger than $315 \mu H_{\odot}$. The hatched histogram is the corrected region counts and the heavy histogram the actual counts of regions.

\section{ORIENTATION OF MAGNETIC AXES}

The orientation of the magnetic axis of a bipolar region, defined as the angle of a line between the centroids of a region's two poles and the equator, was measured in eight $45^{\circ}$ wide bins at the time of maximum development, i.e. when the emergence of magnetic flux ceased. The orientation of regions as proper or reversed is determined according to the Hale Polarity Law for cycle membership and hemisphere.

As shown in Figure 3, regions in every size range, including ephemeral regions, have a preference for a low-inclination, proper orientation. The fraction of regions in the proper orientation bins, however, decreases from $95 \%$ for regions with areas greater than $510 \mu \mathrm{H}_{\odot}$ to $56 \%$ for ephemeral regions (areas 5-120 $\left.\mu \mathrm{H}_{\odot}\right)$. The dispersion in orientation increases with decreasing region size and also increases at greater distances from the average region latitude. For every size range including ephemeral regions, there is a slight preference for reversed regions to have a low-inclination orientation. 


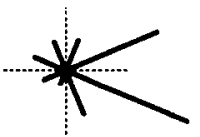

$120-315$

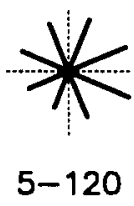

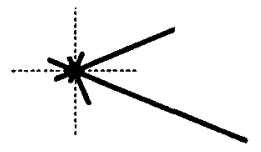

$315-510$

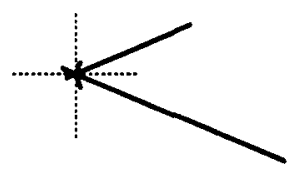

$510-3550$

Fig. 3. Orientations, referenced to the northern hemisphere, at the time of maximum development are shown for regions in four area ranges, indicated at the bottom in $\mu \mathrm{H}_{\odot}$. The vertical dotted line separates proper (to the right) and reversed (left) orientations, and the horizontal line separates orientations where the region's leader is equatorward (below the line) or poleward of the follower (above).

\section{CONCLUSIONS}

The comparison of several properties of active regions and of ephemeral regions leads to the conclusion that ephemeral regions are the small scale end of a wide size spectrum of active regions. The size distribution of ephemeral regions is consistent with and extends smoothly from that of active regions. The latitude distribution of ephemeral regions indicates that they preferentially emerge near the average latitude of larger active regions and are distributed over a wide latitude range. This continues the systematic widening of the latitudinal extent of smaller regions with decreasing area. Though ephemeral regions have a large dispersion in region orientation, they still exhibit a preferential orientation consistent with the Hale Polarity Law.

\section{ACKNOWLEDGEMENTS}

I wish to express my appreciation to Drs. C. Zwaan and R. Howard, for many helpful and informative discussions. This work was funded under contract Air Force Contract AFOSR-90-0006.

\section{REFERENCES}

Harvey, K. L. 1993, submitted to Solar Phys.

Harvey, K. L. and Zwaan, C. 1993, submitted to Solar Phys. 